\title{
The impact of work rest scheduling for prolonged standing activity
}

\author{
Ismail Abdul RAHMAN ${ }^{1 *}$, Norlizaa MOHAMAD ${ }^{2}$, Jafri Mohd ROHANI ${ }^{2}$ and Raemy Md ZEIN ${ }^{1}$ \\ ${ }^{1}$ Ergonomics Excellence Centre, National Institute of Occupational Safety and Health (NIOSH), Malaysia \\ ${ }^{2}$ Faculty of Mechanical Engineering, Universiti Teknologi Malaysia, Malaysia
}

Received February 21, 2018 and accepted September 7, 2018

Published online in J-STAGE September 13, 2018

\begin{abstract}
Prolonged standing can cause discomfort on the body of the workers and can lead to injury and occupational disease. One of the ergonomic intervention is through improving the workrest scheduling. The purpose of this study are to identify the fatigue level from the perception of the worker and to investigate the impact of the work-rest scheduling to the standing workers for $12 \mathrm{~h}$ working time with a different gender. This study involved two methods which are self-assessment of the worker and direct measurement by using electromyography (EMG). For self-assessment, 80 workers have been interviewed using questionnaire in order to identify the fatigue level. For direct measurement, EMG was attached to the 15 selected workers at their respective leg and lower back to analyse the muscle efforts. In terms of perception, the results show the discomfort and fatigue level at the lower body region in the following order as foot ankle, lower back and leg. There is a significant difference between gender on discomfort pain for foot ankle and leg. The results show short frequent break by $10 \mathrm{~min}$ can reduce the fatigue at the leg and infrequent long break is preferable in order to reduce the fatigue at the lower back. In conclusion, it was found that prolonged standing affect the muscle fatigue and discomfort especially lower extremities such as foot ankle, lower back and leg. Besides that, different type of work rest scheduling and gender have significant result towards the muscle fatigue development.
\end{abstract}

Key words: Prolonged standing, Electronics, Muscle fatigue, Discomfort pain, Work rest scheduling

\section{Introduction}

Industrial sector provides more opportunity in job and career development. By increasing the number of the industrial sector directly increase the number of the injuries and occupational diseases in the real life. Without most of the people realized the occupational disease and injuries directed increase the medical cost and loss of the productivity. Based on previous study conducted, one of the factor workplace accident is stress and fatigue ${ }^{1)}$. Prolonged

*To whom correspondence should be addressed.

E-mail: ismailabdulrahman.niosh@gmail.com

(C)2018 National Institute of Occupational Safety and Health standing is one of the common activities in the industrial sector as the task requirement. However, lack of awareness among employer regarding this issue as they believe the break given is sufficient for the employees without taken into consideration of the workload as long as fulfill the target of the company.

One of the methods to reduces the muscles fatigue or pain development in prolonged standing is by administration control, which is job rotation or improve the design of work-rest schedule. As we noticed most of the company having similar break times by neglected the environment of the work or task performance. By improving the workrest schedule without any cost, this helps the workers to reduce the muscle fatigue and improve the health. 
Prolonged standing activity facing by the operators can cause fatigue or discomfort that lead to absenteeism, low productivity, and medical cost. Work-rest schedule is one of the improvement can be applied in reducing the fatigue for prolonged standing and considering the gender of the operators. In general, gender brings significant effect toward work capacity. There are study and research that show women are more resistant to fatigue compared to the $\operatorname{man}^{2}$. Through that research it can be concludes sex differences can effects the muscle fatigue development.

The purpose of this study was to identify the current situation of fatigue level for prolonged standing activity in the industrial workplace of electronic industry, Malaysia. The aims of this study are to analyses the muscle activity for prolonged standing with different work-rest setting and the influences of the gender and towards muscle fatigue. Besides that, at the end of this study, it will recommend a work-rest schedule to reduce fatigue and improve body comfort. The results from this study it can help or become guidelines in develop suitable work-rest schedule for the improvement of the productivity and reduce occupational injuries in the industry.

\section{Subjects and Methods}

This study involves two methods. Firstly, the interviews were conducted among the operator measure assembly line workers' fatigue and discomfort levels. Secondly, the data were collected through the experiment.

\section{Subjects/participants}

This study will involve 10 females and 10 males workers for the experimental. However, due to the several issues only 8 females and 7 males involved and can commit to the end of the experiment. In order to compare the group of the respondent, the health status and experience of the word load should be discernible. Subjects should be considered the representative of the overall population in the company. All subjects have specifically informed regarding the experiment and informed consent have been obtained from subjects. The research and methodology have been review and approved by NIOSH Malaysia Research Board.

\section{Interview survey by questionnaires}

The study based on the questionnaires survey by an interview the operator to measure assembly line workers' fatigue and discomfort levels. Each of the operators is interviewed individually without line leader and top management monitoring. This can reduce the variability of the result and increase the reliability of this study. Subjects were requested to provide demographic information including age, height, weight, and job tenure.

The study group is in electronic industry that represents one of the biggest industries in Malaysia. The respondent is 80 workers performed prolonged standing task are distributed equally between male and female. The study group involved the operators at least 6 months working experience in the company. The subjects of this study were full-time employees working first shift working from 7 am to $7 \mathrm{pm}$. This study used the questionnaire adopted from Halim et al. and some modifications have been done according to the research question and objectives ${ }^{3)}$.

\section{Direct technical measurement}

During the experiment conducted, the participant will perform the task naturally and will receive information earlier regarding the rest time adjustment. The data are collects by using EMG equipment at three different sessions in a day. The three sessions can be divided into the morning session, afternoon, and end of the working time. The studied by Halim et al., they revealed that the longest average time of fatigue at all muscles (six type of lower muscle) in morning session, middle of the workday, and end of workday shows the slight different value for about 40 min per session ${ }^{4}$. This can be recommended that the formation of muscle fatigue will be possible to be obtained during these 40 min working an hour at each session. Therefore, the data will be taken at least every $40 \mathrm{~min}$ of each working sessions during working time.

These measurements are analyzing the body muscles of the subject while performing the task or activity. This measurement analyzes subject's body muscle efforts when performing the work. The electromyography EMG model Biomonitor ME6000 are used to record, store and analyze all data regarding muscle activity of each subject or operators. In EMG measurement, the muscle selections for this study are as shown in Fig. 1.

a) Left/Right erector spinae (lower back)

b) Left/Right tibialis anterior (anterior leg)

c) Left/Right gastrocnemius (posterior leg)

By designing these two types of the work rest setting, two different activity-rest schedules with the same amount of total break time are suggested as shown in Fig. 2. The setting 1 is referred to the actual condition of the workrest in the company. For the setting 2, this study decides to reduce the micro breaks to $10 \mathrm{~min}$ with three difference break time in morning and evening whereby for lunch it 


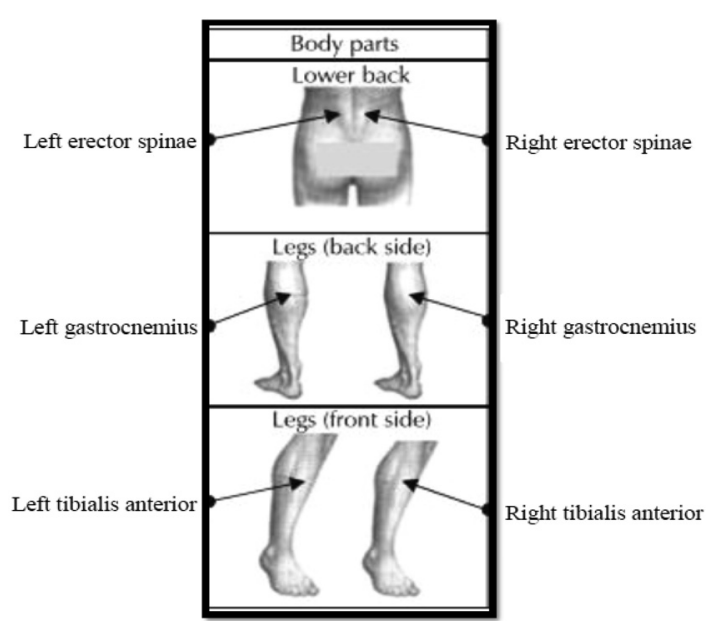

Fig. 1. Location of measurement.

will be maintained as usual.in order to avoid any issues for the production line process, the total break for the setting 2 follows the actual condition where the total break is $90 \mathrm{~min}$.

The result from the questionnaire interview will be analyzed by descriptive statistics as well as the result for experimental. All the variables in the experimental conditions will be repeated using analysis of variance (ANOVA) by performing design of experiment (DOE) by conducting full factorial design. The relationship of the perception between genders towards discomfort body parts is analyzed by using $\chi^{2}$.

\section{Results}

In this study, 85 workers are selected to be interviewed; however, five subjects were eliminated due to the lack of the working experience in the company (less than 6 months). The 80 workers are distributed equally between males and females. The demographic information is summarized in Table 1. The subjects' ages ranged from 19 to $52 \mathrm{yr}$ old with a mean age of $30 \mathrm{yr}$. The height among subjects ranged from $137.10 \mathrm{~cm}$ to $182.30 \mathrm{~cm}$ with a mean height of $159.84 \mathrm{~cm}$. Subjects' weights ranged from $35 \mathrm{~kg}$ to $86 \mathrm{~kg}$. The job tenure of subjects performed prolonged standing task position ranged from 6 months to 228 months with a mean of 59.6 months.

\section{Perception of discomfort pain and fatigue on the body parts}

The ratings of discomfort pain from the body part are explicated in Table 2. The higher number of mean shows that the severity of the discomfort and pain on the body parts is high $(0=$ no fatigue to $4=$ for extreme fatigue). The highest

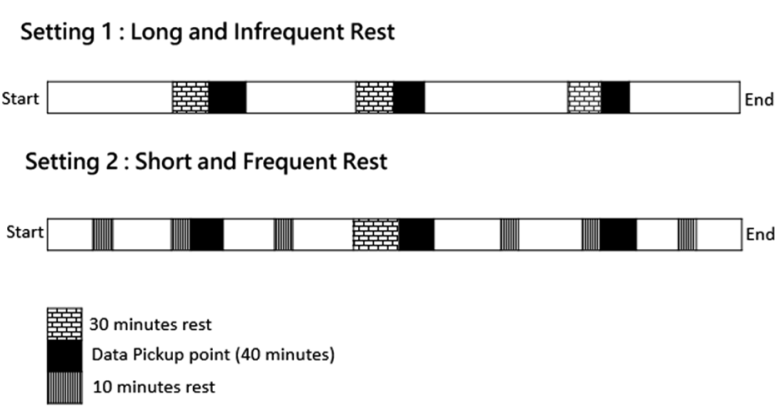

Fig. 2. Work rest scheduling setting.

Table 1. Demographic information $(\mathrm{N}=\mathbf{8 0})$

\begin{tabular}{lccc}
\hline \multicolumn{1}{c}{ Demographic information } & Min & Max & Mean \\
\hline Age & 19.00 & 52.00 & 29.86 \\
Height (cm) & 137.10 & 182.30 & 159.84 \\
Weight (kg) & 35.00 & 86.00 & 59.39 \\
Working experienced (months) & 6.00 & 228.00 & 59.55 \\
Body Mass Index (BMI) & 18.6 & 25.9 & 23.2 \\
\hline
\end{tabular}

mean ratings for discomfort pain are foot ankle (2.7875), lower back (2.4750) and leg (2.0250). The thigh and knee perceived least severity on discomfort pain among the workers on lower extremities. For the categories of upper extremities, shoulder (1.2750) and upper back (1.4250) shows the high rating for the discomfort. This is may be due to the task are performed by the worker by loading and unloading the workload and reaching the goods.

From the result, it clearly indicates the standard deviation is higher than the means except for the lower back, leg and foot ankle. This shows the statistical dispersion of the discomfort rating is widely apart from the means. This also shows the severity of the pain for each of the workers has an enormous difference in terms of perception.

\section{Relationship of gender towards discomfort body}

In order to determine the correlational strength between demographic information and body part discomfort and pain, statistical analysis was computed by using $\chi^{2}$ correlation. The result is shown in Table 3 for analysis of correlations $(p<0.05)$ between gender, age and job tenure for the discomfort on the body parts.

From Tables 4 and 5, several correlations between age and gender towards discomfort body part can be evaluated. Gender was most strongly correlated with discomfort on leg and foot ankle while standing for prolonged periods of time.

Table 4 shows the mean and standard deviation of the difference gender towards discomfort leg. For the female, 
Table 2. Result of discomfort pain and fatigue

\begin{tabular}{lcclcc}
\hline \multicolumn{1}{c}{ Body parts } & Mean & Std. Deviation & Body parts & Mean & Std. Deviation \\
\hline Neck & 0.5250 & 1.1904 & Wrist & 0.3375 & 0.8993 \\
Shoulder & 1.2750 & 1.5008 & Lower back & 2.4750 & 1.8209 \\
Upper back & 1.4250 & 1.4476 & Thigh & 0.4125 & 1.0518 \\
Forearm & 0.6625 & 1.2319 & Knee & 0.7875 & 1.3566 \\
Elbow & 0.2750 & 0.9137 & Leg & 2.0250 & 1.3499 \\
Lower arm & 0.1000 & 0.4666 & Foot ankle & 2.7875 & 1.6512 \\
\hline
\end{tabular}

Table 3. Demographic relationship and body parts discomfort

\begin{tabular}{lclc}
\hline $\begin{array}{l}\text { Area of fatigue/ } \\
\text { Discomfort pain }\end{array}$ & $p$ & $\begin{array}{l}\text { Area of fatigue/ } \\
\text { Discomfort pain }\end{array}$ & $p$ \\
\hline Neck & 0.072 & Wrist & 0.184 \\
Shoulder & 0.029 & Lower back & 0.254 \\
Upper back & 0.273 & Thigh & 0.558 \\
Forearm & 0.726 & Knee & 0.302 \\
Elbow & 0.640 & Leg & 0.020 \\
Lower arm & 0.256 & Foot ankle & 0.000 \\
\hline
\end{tabular}

$p$ : significant 2 tailed $(p<0.05)$.
Table 4. Discomfort leg $(\mathrm{N}=80)$

\begin{tabular}{lccc}
\hline \multicolumn{1}{c}{ Gender } & Mean & Std. Deviation & $\mathrm{t}$ \\
\hline Male & 1.4750 & 1.4320 & \\
Female & 2.5750 & 1.0099 & -3.970 \\
\hline
\end{tabular}

Table 5. Discomfort foot ankle ( $\mathrm{N}=\mathbf{8 0})$

\begin{tabular}{lccc}
\hline \multicolumn{1}{c}{ Gender } & Mean & Std. Deviation & \multicolumn{1}{c}{$\mathrm{t}$} \\
\hline Male & 2.400 & 2.0102 & -2.146 \\
Female & 3.175 & 1.0833 & \\
\hline
\end{tabular}

it has highest mean compared to the male.This can conclude the discomfort pain and fatigue mostly occurs in female compared to the male. The result in Table 5 shows female having discomfort pain and fatigue on the foot ankle compared to male.

\section{Design of experiment}

As this study will be conducted the experimental study on the lower extremities for the lower back and leg, the perception of the workers towards discomfort of the body parts also been asked. The results are shown in Fig. 3. The results shows the most fatigue area at the lower extremities is leg back side followed by lower back and leg front side.

For the design of the experiment, it involved 20 workers randomly selected from the production line by the top management. The 20 workers divided into 10 male workers and 10 female workers. However, due to some issue, only 15 operators which are 7 males and 8 females can be committed to the end of the experiment. In this experiment, it involved three muscles with six different areas to be attached with the EMG. For the result, only five area of the muscle can be analyzed due to the error of the probe at the muscle number six which is lower back right side.

\section{Muscle efforts analysis}

As this experiment are conducted in two difference work-rest and difference gender, each of the muscle efforts data of each muscle will be evaluated based on the trend. It was believed higher muscle efforts were used the higher is the muscle fatigue to be developed.

From Fig. 4, it shows the six difference muscle at the actual work-rest condition in the company. It clearly illustrates at the beginning of the day, it required high muscle effort and gradually decrease as from morning to mid-day and shows small reduction when reaching evening session. However, there is slightly increase for muscle erector spinae for left and right side which is lower back at the end of the day.

Figure 4 also shows the higher muscle efforts are at the lower back followed by gastrocnemius muscle and lastly tibialis anterior muscle. Figure 5 indicates the results for female workers in the company. There is gradually decrease from the morning to the evening session after the three breaks with 30 min of time. The result also shows only gastrocnemius muscle on the left side shows some increment and it is about $2 \%$ from the mid-day session.

When comparing the actual condition between two difference genders, it shows the males worker required high muscle effort at the lower back compared to the females workers. This is may be due the task requirement is different and slightly heavy task compared to females. However, when comparing the muscle efforts for the leg, front, and back side, it shows females suffer from fatigue compared to the male workers. The muscle effort for females is 


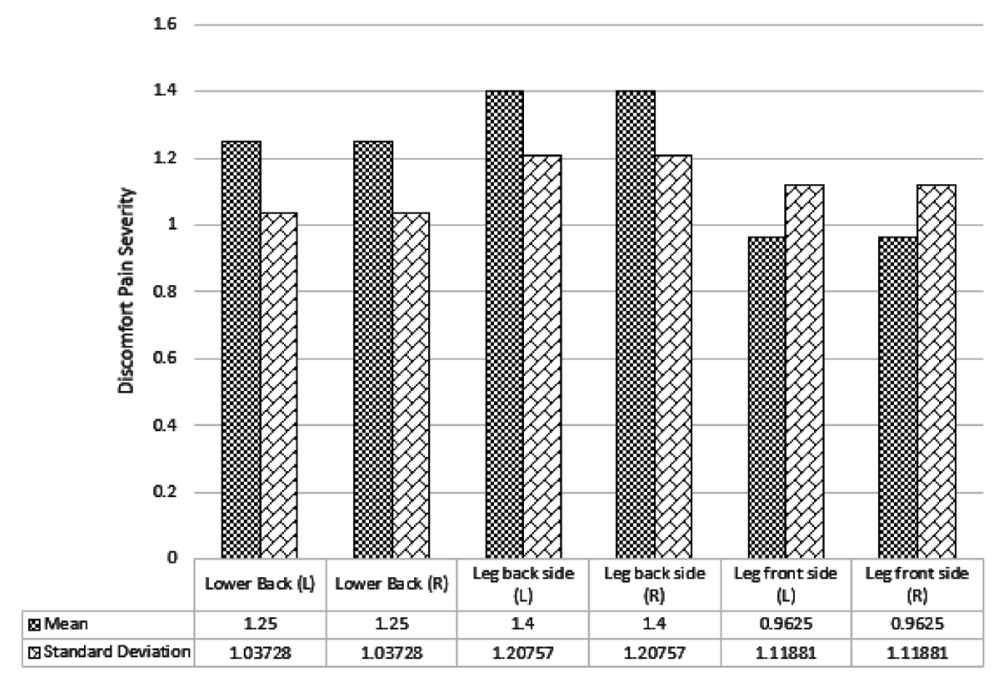

Fig. 3. Result of perception on lower extremities.

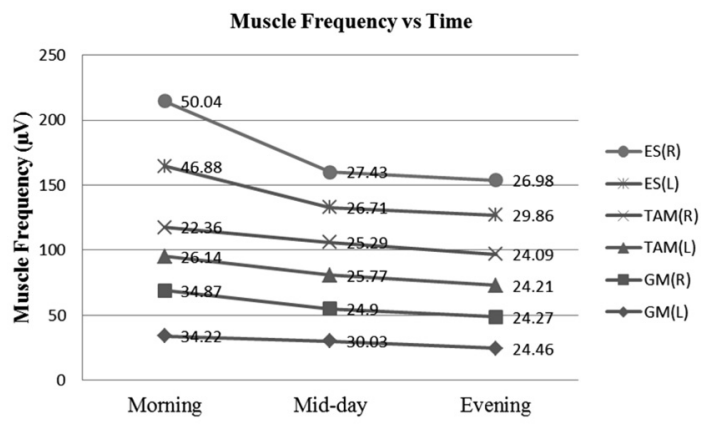

Fig. 4. Muscle frequency vs. time (Males-setting 1).

at least $30 \%$ of the muscle effort for males. This can be supported the result for the perception where the females operators having more discomfort pain and fatigue in the leg compared to males operators.

By changing the setting of short frequent work-rest, the result will be demonstrated in the Fig. 6 for males and Fig. 7 for females' operators. Nevertheless, in this setting there is no evaluation for the muscle 6 as the probe cannot be detected the muscle efforts. From the males result, it shows all the muscle is shown some reduction of the muscle efforts from the actual condition even the trend is increasing at the end of the day. For the erector spinae on the left side, it changed from 46.88 in the actual condition to the 33.83 in the setting 2 in the morning session. For the mid-day and evening session, it shows the increment of the muscle effort compared to the actual condition.

For the tibialis anterior, it also shows the decrement from the actual condition from morning to the midday but in the evening, the muscle effort for both sides is different.

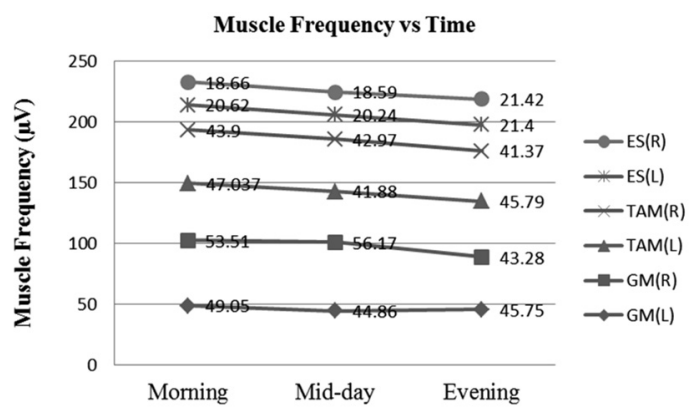

Fig. 5. Muscle frequency vs. time (female-setting 1).

At the right side, the muscle effort requires high than first setting whereby for left side, there id reduction for the actual working work rest. The similar result obtained with tibialis anterior on the right side ad bot side of the gastrocnemius muscle shows the same trend. This can conclude the muscle may be reduced at the setting 2 but at the end of the day, the muscle effort slightly increases compared to the actual working work-rest.

In Fig. 7, as mentioned earlier, it illustrates the result for the female operators in setting 2 conditions. The trends of each muscle are almost similar. In this setting 2 , it most of the muscle shows decrement of the muscle efforts compared to the actual working condition except for erector spinae on the left side. From the result, the erector spinae are required high muscle effort in this setting increase about $87 \%$ in the morning, $63 \%$ for mid-day and $39 \%$ from the actual condition in the evening. The trends for the increment in the evening at others four muscle still shows the reduction compared to the actual working condition. 


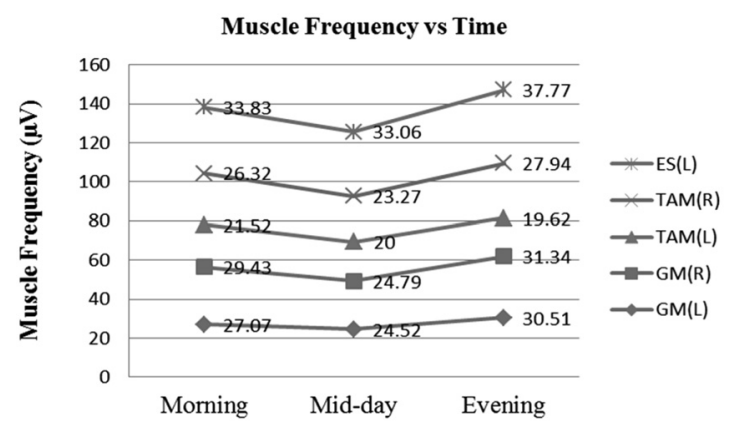

Fig. 6. Muscle frequency vs. time (Males-setting 2).

By comparing both of the results from the setting 2, it can be seen the trend is increasing at the end of the working day even at the beginning of the day it shows the reduction of the muscle effort until mid-day. However, for evening session even increase but the muscle efforts are reduce compared to the actual working condition. There is an exception for the muscle erector spinae (L) as the muscle effort is an increase in this setting. By comparing between gender, females shows high reduction number of the muscle effort compared to the males working in this setting 2 conditions.

Additional analysis is to investigate the relationship between factors of gender and work-rest setting by statistical test through the design of the experiment. The design of the experiment was carried out by two factors at two level are conducted by using Minitab 15, after the data screening in order to know the effects of the muscle fatigue by the factor of gender and work-rest schedule as stated for long-infrequent and short frequent work-rest. A full factorial design with low level and high level was applied for planning the experiment involving muscle fatigue as the response for analysis as Table 6 . Moreover, the selected experimental design is $2^{2}$ full factorial designs by nine replicates were obtained to investigate the muscle fatigue. As the measurement by using EMG probe, the muscle frequency is measured in a unit of microvolt $(\mu \mathrm{V})$.

For leg back side muscle also known as Gastrocnemius muscle shows the different result even the muscle is same. For the muscle 1 which is leg back side left, the work-rest $(p=0.000)$ is the main factor for the fatigue whereby for leg back side right gender $(p=0.021)$ is the main contributor for $p<0.05$. There is no significant between interaction effect for each muscle when $p<0.05$. Even though no interaction effect, but the interaction plot indicates the short frequent break can reduce the muscle fatigue for female for the muscle leg back side left and leg beck side right, but in the leg beck side right muscle, the frequency of the

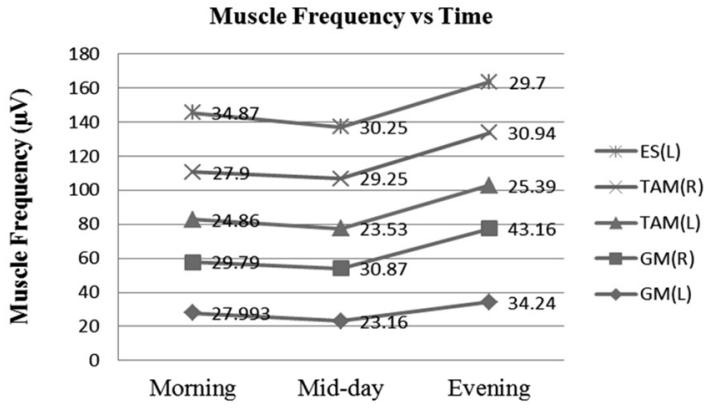

Fig. 7. Muscle frequency vs. time (female-setting 2).

Table 6. Factors and level of process parameters

\begin{tabular}{lcc}
\hline \multicolumn{1}{c}{ Factors } & Low level $(-)$ & High level $(+)$ \\
\hline Gender & Male & Female \\
Work-rest setting & Long infrequent & Short frequent \\
\hline
\end{tabular}

muscle increase for males operator.

For tibialis anterior muscles, both left and right muscle shows a similar result with gender, work-rest setting and the main interaction effect with $p<0.05$. It can be concluded gender and work-rest are significant in causing the muscle fatigue for the tibialis anterior muscle. There is a reduction of the frequency when short frequent break for males and females operators that proves a reduction of fatigue.

Erector spinae muscles or lower back muscle also demonstrates the entire factor which is gender, work-rest and interaction effect are significant when $p<0.05$. This result is similar with the leg front side or tibialis anterior muscles. In terms of the interaction effect long-infrequent work-rest can reduce the fatigue at the lower back. It also clearly can be seen at the frequent short-break the male and female operators having a high number of frequencies of the voltage that can be concluded more fatigue compare to long-infrequent break.

\section{Implication and Discussion}

In this study, through the observation and interview the workers, this study revealed that all the workers worked about $12 \mathrm{~h}$ shift schedules in the production line. Through this $12 \mathrm{~h}$ working, the operators spent about $88 \%$ of standing during performed the task requirement. The longstanding period has far exceeded the threshold limit of the standing time ${ }^{5)}$. This continuous exposure can cause muscle fatigue at the body part.

Through this research by conducting questionnaire survey through an interview, it revealed that leg, lower back, 
and foot ankle are most discomfort pains or fatigue in the body part compared to the other parts. This result also can reach agreement when the study from an Australian reported $91 \%$ of the workers had foot complications and $49 \%$ complained precisely of painful or discomfort on feet $^{6}$. The result of this study also relevance discomfort pain or fatigue on the body such as neck and shoulder. It was documented in the previous study the physiological discomfort experience when a worker who performs processes jobs in a long duration of standing may experience discomfort in the legs, neck and shoulder ${ }^{7)}$.

The variable such as height, weight, age, and job tenure are associated with the discomfort rating on various body parts $^{8)}$. In this study, gender has been discussed with the influence of the discomfort severity level that exposed to the prolonged standing operators based on their perception. Based on gender, there is a strength relation between gender and discomfort on the leg $(p=0.002 ; p<0.05)$ and foot ankle $(p=0.000 ; p<0.05)$. This result can be supported by the previous study reported female significantly more lower-extremity pain than their male colleagues ${ }^{9}$. It was agreed by the other authors that reported female gender was strongly associated with lower extremity pain.

In terms of the perceptions of the workers towards three different area of the muscle, all the workers consider left and right having the same severity of the fatigue. By comparing three different muscles, lower back is the most discomfort area of the body parts compared to the leg at the back side and front side. Based on the gender, females having little fatigue to extreme fatigue higher than males workers with three difference muscle. This shows there is difference perception between genders towards the lower extremity body parts. By comparing with EMG result, it demonstrates the females workers having high muscle effort compared to males worker while conducting the task for prolonged standing. This shows the perception of the workers towards lower extremities muscle is almost similar with the experimental result by using EMG probe.

From the result of the muscle effort analysis, the actual condition shows the trend of decrement from the morning to the evening session of each of the muscle. This is maybe due to the result of the work-rest taken by the operator for $30 \mathrm{~min}$ enough to get a sit and food in order to refresh their energy and slightly reduce the fatigue of the previous activity in the company for each session. In contrast to the 10 min break, through the EMG measurement even it reduces the muscle fatigue but there are small possibilities to allow the operators to eat in the morning and evening session as the operators only depend on the lunch break to eat and re-energized. There are slight differences between left and a right leg based on the EMG result of muscle efforts. These variations may be due to the position of the leg during performing the task at their workstations.

Based on the experiment, the finding indicates short frequent work-rest can reduce the muscle effort compared to the long infrequent break at the both leg muscle (anterior muscle and gastrocnemius muscle). However, it cannot reduce the muscle effort at the lower back (erector spinae muscle). At the lower back, it was suggested for the long infrequent break to reduce fatigue. It can be stated, the 10 min break consider adequate for reducing the muscle fatigue when performed prolonged standing activities in the electronic industry. There is a positive effect on muscle effort measured by EMG when participants had frequent short breaks.

These findings can be justified through the research conducted by the Graf as stated in Kakarot, short breaks (5-10 min each hour) are beneficial for physical as well as mental work resulting in improved physical ${ }^{10)}$ and mental wellbeing and reduced discomfort together with unchanged or even improved performance ${ }^{11)}$. By citing the previous research, Dieen and Vrielink, with respect to muscle fatigue, frequent short breaks are considered more effective than infrequent long breaks ${ }^{12)}$. This proves the short frequent breaks for $10 \mathrm{~min}$ can reduce the muscle fatigue and provides a positive impact.

However, other divergence result shows the indicated that 10-min breaks are insufficient to recover from fatigue, caused by prior work bouts ${ }^{13)}$ however from the findings of this study; it can reduce the muscle fatigue. The research also found long infrequent setting seems more effective in reducing leg swelling however in order for reducing compression on the back spine it was advisable to promote the frequent short rest setting ${ }^{12}$. Nevertheless, in this study, the different result has been obtained. For the back spine, it is preferred to long infrequent breaks instead of short frequent. Besides that, Sheahan et al. also found frequent, short breaks can reduce the low back pain even it is a temporary solution ${ }^{14)}$. This may be due to the workloads or task requirement between electronic and power tiller, poultry operations are different. Overall, as stated by the Tucker, the effectiveness of a particular rest break schedule will be influenced by the nature of work routine and hence will vary from one working environment to the next ${ }^{15}$.

It can be concluded, the perception and experimental obtain almost similar result and gender are one of the factors for muscle fatigue development as well as the work-rest setting. The frequent short breaks seem can reduce the muscle 
fatigue at the leg area not at the lower back of the body and the result shows females required high efforts compared to males workers in the electronic industry in Malaysia.

\section{Conclusion}

From this study, it was found prolonged standing activity in the industrial workplace such as electronic industries cause the muscle fatigue and discomfort body part especially in the lower extremities such as lower back, leg and foot ankle. The perception of the operators towards prolonged standing activities can be justified through the experimental study. By conducting the experiment on the analysis of the muscle effort between two difference work-rest settings, it shows that short frequent breaks for 10 min can reduce the muscle effort at the front and back leg however it is more preferable for lower back pain for long infrequent breaks. By contribute to the research gaps which most of the researcher focus on males operators for work-rest setting experiment, this study includes males and females operator and the result shows there is a significant towards the muscle fatigue development.

\section{Limitations and Future Works}

This study having several limitations such as the EMG probe which is at the short frequent setting the probe cannot detect the lower back on the right side. Thus, it because the lower back for the right side cannot be analyse. Besides that, there is sudden breakdown; stoppage and also resignation of the operator cause the number of the participant reduce from the actual target. This study also not consider the age of the workers.

For the future works, it was suggested to consider the age as the factor for the muscle fatigue as based on the literature age can be one of the important factors. Besides that, it can be suggested to further study for lower back pain with different work-rest setting as this study found long infrequent is better than short frequent which is contradicted from the several literature.

\section{Acknowledgements}

The authors would like to acknowledge National Institute of Occupational Safety and Health (NIOSH) Malaysia for their grant and funding. Also, special thanks to the research team from Faculty of Mechanical Engineering, Universiti Teknologi Malaysia for their knowledge sharing and support. All authors declare that they have no conflicts of interest.

\section{References}

1) Zakaria NH, Mansor N, Abdullah Z (2012) Workplace accident in Malaysia: most common causes and solutions. Business Management Review 2, 75-88.

2) Hicks AL, Kent-Braun J, Ditor DS (2001) Sex differences in human skeletal muscle fatigue. Exerc Sport Sci Rev 29, 109-12. [Medline] [CrossRef]

3) Halim I, Omar AR, Saman AM, Othman I, Ali MA (2010) Development of a questionnaire for prolonged standing jobs at manufacturing industry. Advanc Human Factors, Ergo, Saf in Manfetrg \& Srves Ind, 253-261.

4) Halim I, Omar AR, Saman AM, Othman I (2012) Assessment of muscle fatigue associated with prolonged standing in the workplace. Saf Health Work 3, 31-42. [Medline] [CrossRef]

5) Ahmada N, Taha Z, Eu PL (2006) Energetic requirement, muscle fatigue, and musculoskeletal risk of prolonged standing on female Malaysian operators in the electronic industries: influence of age. Eng e-Transac 1, 47-50.

6) Marr SJ, Quine S (1993) Shoe concerns and foot problems of wearers of safety footwear. Occup Med (Lond) 43, 73-7. [Medline] [CrossRef]

7) Halim I, Omar AR, Saman AM, Othman I (2011) A review on health effects associated with prolonged standing in the industrial workplaces. IJRRAS 8, 14-21.

8) King PM (2002) A comparison of the effects of floor mats and shoe in-soles on standing fatigue. Appl Ergon 33, 477-84. [Medline] [CrossRef]

9) Laperriére E "Le travail de serveuse de restaurant." $\mathrm{PhD}$ diss., Department of Biological Sciences, University of Québec at Montréal, Montréal, QC, 2014.

10) Kakarot N, Mueller F, Bassarak C (2012) Activity-rest schedules in physically demanding work and the variation of responses with age. Ergonomics 55, 282-94. [Medline] [CrossRef]

11) Dababneh AJ, Swanson N, Shell RL (2001) Impact of added rest breaks on the productivity and well being of workers. Ergonomics 44, 164-74. [Medline] [CrossRef]

12) Van Dieën JH, Oude Vrielink HH (1998) Evaluation of work-rest schedules with respect to the effects of postural workload in standing work. Ergonomics 41, 1832-44. [Medline] [CrossRef]

13) Tiwari PS, Gite LP (2006) Evaluation of work-rest schedules during operation of a rotary power tiller. Int J Ind Ergon 36, 203-10. [CrossRef]

14) Sheahan PJ, Diesbourg TL, Fischer SL (2016) The effect of rest break schedule on acute low back pain development in pain and non-pain developers during seated work. Appl Ergon 53 Pt A, 64-70. [Medline] [CrossRef]

15) Tucker $P$ (2003) The impact of rest breaks upon accident risk, fatigue and performance: a review. Work Stress 17, 123-37. [CrossRef] 